\title{
On a generalization of a Hilbert-type integral inequality in the whole plane with a hypergeometric function
}

\author{
Vandanjav Adiyasuren ${ }^{1}$ and Tserendorj Batbold ${ }^{2 *}$
}

\section{"Correspondence:}

tsbatbold@hotmail.com

${ }^{2}$ Institute of Mathematics, National

University of Mongolia, P.O. Box

46A/104, Ulaanbaatar, 14201,

Mongolia

Full list of author information is

available at the end of the article

\begin{abstract}
By introducing some parameters, we establish a generalization of the Hilbert-type integral inequality in the whole plane with the homogeneous kernel of degree $-2 \lambda$ and the best constant factor which involves the hypergeometric function.

MSC: 26D15
\end{abstract}

Keywords: Hilbert's inequality; Hölder's inequality; homogeneous kernel; weight function; equivalent form

\section{Introduction}

If $p>1, \frac{1}{p}+\frac{1}{q}=1$ and $f(x), g(x) \geq 0$ satisfy

$$
0<\int_{0}^{\infty} f^{p}(x) d x<\infty \text { and } 0<\int_{0}^{\infty} g^{q}(x) d x<\infty,
$$

then

$$
\int_{0}^{\infty} \int_{0}^{\infty} \frac{f(x) g(y)}{x+y} d x d y<\frac{\pi}{\sin (\pi / p)}\left\{\int_{0}^{\infty} f^{p}(x) d x\right\}^{\frac{1}{p}}\left\{\int_{0}^{\infty} g^{q}(x) d x\right\}^{\frac{1}{q}},
$$

where the constant factor $\pi /(\sin \pi / p)$ is the best possible. Inequality (1) is called HardyHilbert's inequality [1] and is important in analysis and applications [2].

In 2001, Yang gave an extension of (1) involving beta function as (see [3]):

$$
\begin{aligned}
& \int_{0}^{\infty} \int_{0}^{\infty} \frac{f(x) g(y)}{(x+y)^{\lambda}} d x d y \\
& \quad<B\left(\frac{p+\lambda-2}{p}, \frac{q+\lambda-2}{q}\right)\left\{\int_{0}^{\infty} x^{1-\lambda} f^{p}(x) d x\right\}^{\frac{1}{p}}\left\{\int_{0}^{\infty} x^{1-\lambda} g^{q}(x) d x\right\}^{\frac{1}{q}},
\end{aligned}
$$

where the constant factor $B\left(\frac{p+\lambda-2}{p}, \frac{q+\lambda-2}{q}\right)(\lambda>2-\min \{p, q\})$ is the best possible.

Recently, some new Hilbert-type inequalities in the whole plane have been obtained [4, 5]. Xin and Yang in [5] established the following:

$$
\begin{aligned}
\text { If } p & >1, \frac{1}{p}+\frac{1}{q}=1,|\beta|<1,0<\alpha_{1}<\alpha_{2}<\pi, f, g \geq 0 \text {, satisfy } \\
& 0<\int_{-\infty}^{\infty}|x|^{-p \beta-1} f^{p}(x) d x<\infty \text { and } 0<\int_{-\infty}^{\infty}|y|^{q \beta-1} g^{q}(y) d y<\infty,
\end{aligned}
$$

๑ 2013 Adiyasuren and Batbold; licensee Springer. This is an Open Access article distributed under the terms of the Creative Commons Attribution License (http://creativecommons.org/licenses/by/2.0), which permits unrestricted use, distribution, and reproduction in any medium, provided the original work is properly cited. 
then we have

$$
\begin{aligned}
& \int_{-\infty}^{\infty} \int_{-\infty}^{\infty} \min _{i \in\{1,2\}}\left\{\frac{1}{x^{2}+2 x y \cos \alpha_{i}+y^{2}}\right\} f(x) g(y) d x d y \\
& <k(\beta)\left(\int_{-\infty}^{\infty}|x|^{-p \beta-1} f^{p}(x) d x\right)^{\frac{1}{p}}\left(\int_{-\infty}^{\infty}|y|^{q \beta-1} g^{q}(y) d y\right)^{\frac{1}{q}}
\end{aligned}
$$

and

$$
\begin{aligned}
& \int_{-\infty}^{\infty}|y|^{p(1-\beta)-1}\left(\int_{-\infty}^{\infty} \min _{i \in\{1,2\}}\left\{\frac{1}{x^{2}+2 x y \cos \alpha_{i}+y^{2}}\right\} f(x) d x\right)^{p} d y \\
& \quad<k^{p}(\beta) \int_{-\infty}^{\infty}|x|^{-p \beta-1} f^{p}(x) d x,
\end{aligned}
$$

where the constant factors $k(\beta)=\frac{\pi}{\sin \beta \pi}\left(\frac{\sin \beta \alpha_{1}}{\sin \alpha_{1}}+\frac{\sin \beta\left(\pi-\alpha_{2}\right)}{\sin \alpha_{2}}\right)$ and $k^{p}(\beta)$ are the best possible. Inequalities (3) and (4) are equivalent.

By introducing some parameters, we establish generalizations of inequalities (3) and (4) with the homogeneous kernel of degree $-2 \lambda$ and the best constant factor which involves the hypergeometric function.

\section{Preliminary lemmas}

In order to prove our assertions, we need the following lemmas.

Recall that the hypergeometric function $F(\alpha, \beta ; \gamma ; x)$ is defined [6] by

$$
F(\alpha, \beta ; \gamma ; x)=\sum_{r=0}^{\infty} \frac{(\alpha)_{r}(\beta)_{r}}{(\gamma)_{r}} \frac{x^{r}}{r !}
$$

where $(\alpha)_{r}$ is the Pochhammer symbol defined by

$$
(\alpha)_{r}=\alpha(\alpha+1) \cdots(\alpha+r-1)=\frac{\Gamma(\alpha+r)}{\Gamma(\alpha)} .
$$

It is known the series (5) converges for $|x|<1$ and diverges for $|x|>1$. The hypergeometric function satisfies the integral representation

$$
F(\alpha, \beta ; \gamma ; x)=\frac{\Gamma(\gamma)}{\Gamma(\beta) \Gamma(\gamma-\beta)} \int_{0}^{1} t^{\beta-1}(1-t)^{\gamma-\beta-1}(1-x t)^{-\alpha} d t, \quad \text { if } \gamma>\beta>0 .
$$

Lemma 2.1 (See [7]) Suppose that $a, c>0, b^{2}<a c, 0<\alpha<2 \lambda$. Then we have

$$
\int_{0}^{\infty} \frac{x^{\alpha-1}}{\left(a x^{2}+2 b x+c\right)^{\lambda}} d x=a^{-\frac{\alpha}{2}} c^{\frac{\alpha}{2}-\lambda} B(\alpha, 2 \lambda-\alpha) F\left(\frac{\alpha}{2}, \lambda-\frac{\alpha}{2} ; \lambda+\frac{1}{2} ; 1-\frac{b^{2}}{a c}\right) .
$$

Lemma 2.2 Let $a, c, \lambda>0, b \geq 0,1-2 \lambda<\beta<1$ and $0<\alpha_{1}<\alpha_{2}<\pi$ be real parameters such that $b^{2} \max \left\{\cos ^{2} \alpha_{1}, \cos ^{2}\left(\pi-\alpha_{2}\right)\right\}<a c$. Define the weight functions $\omega(x)$ and $\varpi(y)$ $(x, y \in(-\infty, \infty))$ as follows:

$$
\omega(x):=\int_{-\infty}^{\infty} \min _{i \in\{1,2\}}\left\{\frac{1}{\left(a x^{2}+2 b x y \cos \alpha_{i}+c y^{2}\right)^{\lambda}}\right\} \frac{|x|^{\beta+2 \lambda-1}}{|y|^{\beta}} d y,
$$




$$
\varpi(y):=\int_{-\infty}^{\infty} \min _{i \in\{1,2\}}\left\{\frac{1}{\left(a x^{2}+2 b x y \cos \alpha_{i}+c y^{2}\right)^{\lambda}}\right\} \frac{|y|^{1-\beta}}{|x|^{-\beta-2 \lambda+2}} d x
$$

Then we have $\omega(x)=\varpi(y)=C_{\lambda}(x, y \neq 0)$, where

$$
\begin{aligned}
C_{\lambda}= & a^{\frac{1-\beta}{2}-\lambda} c^{\frac{1-\beta}{2}} B(1-\beta, 2 \lambda+\beta-1) \\
& \times\left[F\left(\frac{1-\beta}{2}, \lambda-\frac{1-\beta}{2} ; \lambda+\frac{1}{2} ; 1-\frac{b^{2} \cos ^{2} \alpha_{1}}{a c}\right)\right. \\
& \left.+F\left(\frac{1-\beta}{2}, \lambda-\frac{1-\beta}{2} ; \lambda+\frac{1}{2} ; 1-\frac{b^{2} \cos ^{2}\left(\pi-\alpha_{2}\right)}{a c}\right)\right] .
\end{aligned}
$$

Proof For $x \in(-\infty, 0)$, setting $u=y / x, u=-y / x$ in the following two integrals, respectively, and using Lemma 2.1, we get

$$
\begin{aligned}
\omega(x)= & \int_{-\infty}^{0} \frac{1}{\left(a x^{2}+2 b x y \cos \alpha_{1}+c y^{2}\right)^{\lambda}} \frac{(-x)^{\beta+2 \lambda-1}}{(-y)^{\beta}} d y \\
& +\int_{0}^{\infty} \frac{1}{\left(a x^{2}+2 b x y \cos \alpha_{2}+c y^{2}\right)^{\lambda}} \frac{(-x)^{\beta+2 \lambda-1}}{y^{\beta}} d y \\
= & \int_{0}^{\infty} \frac{u^{-\beta}}{\left(c u^{2}+2 b u \cos \alpha_{1}+a\right)^{\lambda}} d u+\int_{0}^{\infty} \frac{u^{-\beta}}{\left(c u^{2}+2 b u \cos \left(\pi-\alpha_{2}\right)+a\right)^{\lambda}} d u \\
= & C_{\lambda} .
\end{aligned}
$$

For $x \in(0, \infty)$, setting $u=-y / x, u=y / x$ in the following two integrals, respectively, and using Lemma 2.1, we get

$$
\begin{aligned}
\omega(x)= & \int_{-\infty}^{0} \frac{1}{\left(a x^{2}+2 b x y \cos \alpha_{2}+c y^{2}\right)^{\lambda}} \frac{x^{\beta+2 \lambda-1}}{(-y)^{\beta}} d y \\
& +\int_{0}^{\infty} \frac{1}{\left(a x^{2}+2 b x y \cos \alpha_{1}+c y^{2}\right)^{\lambda}} \frac{x^{\beta+2 \lambda-1}}{y^{\beta}} d y \\
= & \int_{0}^{\infty} \frac{u^{-\beta}}{\left(c u^{2}+2 b u \cos \left(\pi-\alpha_{2}\right)+a\right)^{\lambda}} d u+\int_{0}^{\infty} \frac{u^{-\beta}}{\left(c u^{2}+2 b u \cos \alpha_{1}+a\right)^{\lambda}} d u \\
= & C_{\lambda} .
\end{aligned}
$$

By the same way, we still can find that $\omega(x)=\varpi(y)=C_{\lambda}(x, y \neq 0)$. The lemma is proved.

Lemma 2.3 Let $p$ and $q$ be conjugate parameters with $p>1$, and let $a, c, \lambda>0, b \geq 0,1-$ $2 \lambda<\beta<1,0<\alpha_{1}<\alpha_{2}<\pi$ and $b^{2} \max \left\{\cos ^{2} \alpha_{1}, \cos ^{2}\left(\pi-\alpha_{2}\right)\right\}<a c$, and $f(x)$ be a nonnegative measurable function in $(-\infty, \infty)$, then we have

$$
\begin{aligned}
J & :=\int_{-\infty}^{\infty}|y|^{p(1-\beta)-1}\left(\int_{-\infty}^{\infty} \min _{i \in\{1,2\}}\left\{\frac{1}{\left(a x^{2}+2 b x y \cos \alpha_{i}+c y^{2}\right)^{\lambda}}\right\} f(x) d x\right)^{p} d y \\
& \leq C_{\lambda}^{p} \int_{-\infty}^{\infty}|x|^{-p(\beta+2 \lambda-2)-1} f^{p}(x) d x .
\end{aligned}
$$


Proof By Lemma 2.2 and Hölder's inequality [8], we have

$$
\begin{aligned}
&\left(\int_{-\infty}^{\infty} \min _{i \in\{1,2\}}\left\{\frac{1}{\left(a x^{2}+2 b x y \cos \alpha_{i}+c y^{2}\right)^{\lambda}}\right\} f(x) d x\right)^{p} \\
&= {\left[\int_{-\infty}^{\infty} \min _{i \in\{1,2\}}\left\{\frac{1}{\left(a x^{2}+2 b x y \cos \alpha_{i}+c y^{2}\right)^{\lambda}}\right\}\right.} \\
&\left.\times\left(\frac{|x|^{(-\beta-2 \lambda+2) / q}}{|y|^{\beta / p}} f(x)\right)\left(\frac{|y|^{\beta / p}}{|x|^{(-\beta-2 \lambda+2) / q}}\right) d x\right]^{p} \\
& \leq \int_{-\infty}^{\infty} \min _{i \in\{1,2\}}\left\{\frac{|x|^{(1-p)(\beta+2 \lambda-2)}}{\left(a x^{2}+2 b x y \cos \alpha_{i}+c y^{2}\right)^{\lambda}} f^{p}(x) d x\right. \\
& \times\left(\int_{-\infty}^{\infty} \min _{i \in\{1,2\}}\left\{\frac{1}{\left(a x^{2}+2 b x y \cos \alpha_{i}+c y^{2}\right)^{\lambda}}\right\} \frac{|y|^{(q-1) \beta}}{|x|^{(-\beta-2 \lambda+2)}} d x\right)^{p-1} \\
&= C_{\lambda}^{p-1}|y|^{p(\beta-1)+1} \int_{-\infty}^{\infty} \min _{i \in\{1,2\}}\left\{\frac{1}{\left(a x^{2}+\left.2 b x y\right|^{(1-p)(\beta+2 \lambda-2)}\right.} f^{p}(x) d x .\right.
\end{aligned}
$$

Then by the Fubini theorem, it follows that

$$
\begin{aligned}
J & \leq C_{\lambda}^{p-1} \int_{-\infty}^{\infty}\left[\int_{-\infty}^{\infty} \min _{i \in\{1,2\}}\left\{\frac{1}{\left(a x^{2}+2 b x y \cos \alpha_{i}+c y^{2}\right)^{\lambda}}\right\} \frac{|x|^{(1-p)(\beta+2 \lambda-2)}}{|y|^{\beta}} f^{p}(x) d x\right] d y \\
& =C_{\lambda}^{p-1} \int_{-\infty}^{\infty} \omega(x)|x|^{-p(\beta+2 \lambda-2)-1} f^{p}(x) d x \\
& =C_{\lambda}^{p} \int_{-\infty}^{\infty}|x|^{-p(\beta+2 \lambda-2)-1} f^{p}(x) d x .
\end{aligned}
$$

The lemma is proved.

\section{Main results}

Theorem 3.1 Let $p$ and $q$ be conjugate parameters with $p>1$, and let $a, c, \lambda>0, b \geq 0$, $1-2 \lambda<\beta<1,0<\alpha_{1}<\alpha_{2}<\pi$ and $b^{2} \max \left\{\cos ^{2} \alpha_{1}, \cos ^{2}\left(\pi-\alpha_{2}\right)\right\}<a c$, and $f, g \geq 0$, satisfy $0<\int_{-\infty}^{\infty}|x|^{-p(\beta+2 \lambda-2)-1} f^{p}(x) d x<\infty$ and $0<\int_{-\infty}^{\infty}|y|^{q \beta-1} g^{q}(y) d y<\infty$. Then

$$
\begin{aligned}
I & :=\int_{-\infty}^{\infty} \int_{-\infty}^{\infty} \min _{i \in\{1,2\}}\left\{\frac{1}{\left(a x^{2}+2 b x y \cos \alpha_{i}+c y^{2}\right)^{\lambda}}\right\} f(x) g(y) d x d y \\
& <C_{\lambda}\left(\int_{-\infty}^{\infty}|x|^{-p(\beta+2 \lambda-2)-1} f^{p}(x) d x\right)^{1 / p}\left(\int_{-\infty}^{\infty}|y|^{q \beta-1} g^{q}(y) d y\right)^{1 / q},
\end{aligned}
$$

and

$$
\begin{aligned}
J & =\int_{-\infty}^{\infty}|y|^{p(1-\beta)-1}\left(\int_{-\infty}^{\infty} \min _{i \in\{1,2\}}\left\{\frac{1}{\left(a x^{2}+2 b x y \cos \alpha_{i}+c y^{2}\right)^{\lambda}}\right\} f(x) d x\right)^{p} d y \\
& <C_{\lambda}^{p} \int_{-\infty}^{\infty}|x|^{-p(\beta+2 \lambda-2)-1} f^{p}(x) d x,
\end{aligned}
$$

where the constant factors $C_{\lambda}$ and $C_{\lambda}^{p}$ are the best possible and $C_{\lambda}$ is defined in Lemma 2.2. Inequalities (8) and (9) are equivalent. 
Proof If (7) takes the form of the equality for a $y \in(-\infty, 0) \cup(0, \infty)$, then there exist constants $A$ and $B$ such that they are not all zero, and

$$
A \frac{|x|^{(1-p)(\beta+2 \lambda-2)}}{|y|^{\beta}} f^{p}(x)=B \frac{|y|^{(q-1) \beta}}{|x|^{(-\beta-2 \lambda+2)}} \quad \text { a.e. in }(-\infty, \infty) \times(-\infty, \infty) .
$$

Hence, there exists a constant $K$ such that

$$
A|x|^{p(\beta+2 \lambda-2)} f^{p}(x)=B|y|^{q \beta}=K \quad \text { a.e. in }(-\infty, \infty) \times(-\infty, \infty) .
$$

We suppose $A \neq 0$ (otherwise $B=A=0$ ). Then $|x|^{p(\beta+2 \lambda-2)-1} f^{p}(x)=K /(A|x|)$ a.e. in $(-\infty, \infty)$, which contradicts the fact that $0<\int_{-\infty}^{\infty}|x|^{-p(\beta+2 \lambda-2)-1} f^{p}(x) d x<\infty$. Hence, (7) takes the form of a strict inequality, so does (6), and we have (9).

By Hölder's inequality [8], we have

$$
\begin{aligned}
I= & \int_{-\infty}^{\infty}\left(|y|^{1 / q-\beta} \int_{-\infty}^{\infty} \min _{i \in\{1,2\}}\left\{\frac{1}{\left(a x^{2}+2 b x y \cos \alpha_{i}+c y^{2}\right)^{\lambda}}\right\} f(x) d x\right) \\
& \times\left(|y|^{\beta-1 / q} g(y)\right) d y \\
\leq & J^{1 / p}\left(\int_{-\infty}^{\infty}|y|^{\beta-1} g^{q}(y) d y\right)^{1 / q} .
\end{aligned}
$$

By (9), we have (8). On the other hand, suppose that (8) is valid. Set

$$
g(y)=|y|^{p(1-\beta)-1}\left(\int_{-\infty}^{\infty} \min _{i \in\{1,2\}}\left\{\frac{1}{\left(a x^{2}+2 b x y \cos \alpha_{i}+c y^{2}\right)^{\lambda}}\right\} f(x) d x\right)^{p-1},
$$

then it follows $J=\int_{-\infty}^{\infty}|y|^{q \beta-1} g^{q}(y) d y$. By (6), we have $J<\infty$. If $J=0$, then (9) is obviously valid. If $0<J<\infty$, then by (8), we obtain

$$
\begin{aligned}
0 & <\int_{-\infty}^{\infty}|y|^{q \beta-1} g^{q}(y) d y=J=I \\
& <C_{\lambda}\left(\int_{-\infty}^{\infty}|x|^{-p(\beta+2 \lambda-2)-1} f^{p}(x) d x\right)^{1 / p}\left(\int_{-\infty}^{\infty}|y|^{q \beta-1} g^{q}(y) d y\right)^{1 / q},
\end{aligned}
$$

and

$$
\begin{aligned}
J^{1 / p} & =\left(\int_{-\infty}^{\infty}|y|^{q \beta-1} g^{q}(y) d y\right)^{1 / p} \\
& <C_{\lambda}\left(\int_{-\infty}^{\infty}|x|^{-p(\beta+2 \lambda-2)-1} f^{p}(x) d x\right)^{1 / p} .
\end{aligned}
$$

Hence, we have (9), which is equivalent to (8).

For $\varepsilon>0$, define functions $\widetilde{f}(x), \widetilde{g}(y)$ as follows:

$$
\tilde{f}(x):= \begin{cases}x^{(\beta+2 \lambda-2)-2 \varepsilon / p}, & x \in(1, \infty), \\ 0, & x \in[-1,1], \\ (-x)^{(\beta+2 \lambda-2)-2 \varepsilon / p}, & x \in(-\infty,-1),\end{cases}
$$




$$
\tilde{g}(y):= \begin{cases}y^{-\beta-2 \varepsilon / q}, & y \in(1, \infty), \\ 0, & y \in[-1,1], \\ (-y)^{-\beta-2 \varepsilon / q}, & y \in(-\infty,-1) .\end{cases}
$$

Then

$$
\widetilde{L}:=\left(\int_{-\infty}^{\infty}|x|^{-p(\beta+2 \lambda-2)-1} \widetilde{f}^{p}(x) d x\right)^{1 / p}\left(\int_{-\infty}^{\infty}|y|^{q \beta-1} \widetilde{g}^{q}(y) d y\right)^{1 / q}=\frac{1}{\varepsilon}
$$

and

$$
\begin{aligned}
\widetilde{I} & :=\int_{-\infty}^{\infty} \int_{-\infty}^{\infty} \min _{i \in\{1,2\}}\left\{\frac{1}{\left(a x^{2}+2 b x y \cos \alpha_{i}+c y^{2}\right)^{\lambda}}\right\} \tilde{f}(x) \widetilde{g}(y) d x d y \\
& =I_{1}+I_{2}+I_{3}+I_{4},
\end{aligned}
$$

where

$$
\begin{aligned}
& I_{1}:=\int_{-\infty}^{-1}(-x)^{(\beta+2 \lambda-2)-2 \varepsilon / p}\left[\int_{-\infty}^{-1} \frac{(-y)^{-\beta-2 \varepsilon / q}}{\left(a x^{2}+2 b x y \cos \alpha_{1}+c y^{2}\right)^{\lambda}} d y\right] d x, \\
& I_{2}:=\int_{-\infty}^{-1}(-x)^{(\beta+2 \lambda-2)-2 \varepsilon / p}\left[\int_{1}^{\infty} \frac{y^{-\beta-2 \varepsilon / q}}{\left(a x^{2}+2 b x y \cos \alpha_{2}+c y^{2}\right)^{\lambda}} d y\right] d x, \\
& I_{3}:=\int_{1}^{\infty} x^{(\beta+2 \lambda-2)-2 \varepsilon / p}\left[\int_{-\infty}^{-1} \frac{(-y)^{-\beta-2 \varepsilon / q}}{\left(a x^{2}+2 b x y \cos \alpha_{2}+c y^{2}\right)^{\lambda}} d y\right] d x
\end{aligned}
$$

and

$$
I_{4}:=\int_{1}^{\infty} x^{(\beta+2 \lambda-2)-2 \varepsilon / p}\left[\int_{1}^{\infty} \frac{y^{-\beta-2 \varepsilon / q}}{\left(a x^{2}+2 b x y \cos \alpha_{1}+c y^{2}\right)^{\lambda}} d y\right] d x
$$

Taking $u=y / x$, by the Fubini theorem, we obtain

$$
\begin{aligned}
I_{1}= & I_{4}=\int_{1}^{\infty} x^{-1-2 \varepsilon} \int_{1 / x}^{\infty} \frac{u^{-\beta-2 \varepsilon / q}}{\left(c u^{2}+2 b u \cos \alpha_{1}+a\right)^{\lambda}} d u d x \\
= & \int_{1}^{\infty} x^{-1-2 \varepsilon}\left(\int_{1 / x}^{1} \frac{u^{-\beta-2 \varepsilon / q}}{\left(c u^{2}+2 b u \cos \alpha_{1}+a\right)^{\lambda}} d u+\int_{1}^{\infty} \frac{u^{-\beta-2 \varepsilon / q}}{\left(c u^{2}+2 b u \cos \alpha_{1}+a\right)^{\lambda}} d u\right) d x \\
= & \int_{0}^{1}\left(\int_{1 / u}^{\infty} x^{-1-2 \varepsilon} d x\right) \frac{u^{-\beta-2 \varepsilon / q}}{\left(c u^{2}+2 b u \cos \alpha_{1}+a\right)^{\lambda}} d u \\
& +\frac{1}{2 \varepsilon} \int_{1}^{\infty} \frac{u^{-\beta-2 \varepsilon / q}}{\left(c u^{2}+2 b u \cos \alpha_{1}+a\right)^{\lambda}} d u \\
= & \frac{1}{2 \varepsilon}\left(\int_{0}^{1} \frac{u^{-\beta-2 \varepsilon / q}}{\left(c u^{2}+2 b u \cos \alpha_{1}+a\right)^{\lambda}} d u+\int_{1}^{\infty} \frac{u^{-\beta-2 \varepsilon / q}}{\left(c u^{2}+2 b u \cos \alpha_{1}+a\right)^{\lambda}} d u\right),
\end{aligned}
$$

and

$$
I_{2}=I_{3}=\frac{1}{2 \varepsilon}\left(\int_{0}^{1} \frac{u^{-\beta-2 \varepsilon / q}}{\left(c u^{2}-2 b u \cos \alpha_{2}+a\right)^{\lambda}} d u+\int_{1}^{\infty} \frac{u^{-\beta-2 \varepsilon / q}}{\left(c u^{2}-2 b u \cos \alpha_{2}+a\right)^{\lambda}} d u\right)
$$


In view of the above results, if the constant factor $C_{\lambda}$ in (8) is not the best possible, then there exists a positive number $\widetilde{C}$ with $\widetilde{C}<C_{\lambda}$ such that

$$
\begin{aligned}
& \int_{0}^{1} \frac{u^{-\beta-2 \varepsilon / q}}{\left(c u^{2}+2 b u \cos \alpha_{1}+a\right)^{\lambda}} d u+\int_{1}^{\infty} \frac{u^{-\beta-2 \varepsilon / q}}{\left(c u^{2}+2 b u \cos \alpha_{1}+a\right)^{\lambda}} d u \\
& \quad+\int_{0}^{1} \frac{u^{-\beta-2 \varepsilon / q}}{\left(c u^{2}-2 b u \cos \alpha_{2}+a\right)^{\lambda}} d u+\int_{1}^{\infty} \frac{u^{-\beta-2 \varepsilon / q}}{\left(c u^{2}-2 b u \cos \alpha_{2}+a\right)^{\lambda}} d u \\
&=\varepsilon \widetilde{I}<\varepsilon \widetilde{C} \cdot \widetilde{L}=\widetilde{C} .
\end{aligned}
$$

By the Fatou lemma and (11), we have

$$
\begin{aligned}
C_{\lambda}= & \int_{0}^{\infty} \frac{u^{-\beta}}{\left(c u^{2}+2 b u \cos \alpha_{1}+a\right)^{\lambda}} d u+\int_{0}^{\infty} \frac{u^{-\beta}}{\left(c u^{2}-2 b u \cos \alpha_{2}+a\right)^{\lambda}} d u \\
= & \int_{0}^{1} \lim _{\varepsilon \rightarrow 0^{+}} \frac{u^{-\beta-2 \varepsilon / q}}{\left(c u^{2}+2 b u \cos \alpha_{1}+a\right)^{\lambda}} d u+\int_{1}^{\infty} \lim _{\varepsilon \rightarrow 0^{+}} \frac{u^{-\beta-2 \varepsilon / q}}{\left(c u^{2}+2 b u \cos \alpha_{1}+a\right)^{\lambda}} d u \\
& +\int_{0}^{1} \lim _{\varepsilon \rightarrow 0^{+}} \frac{u^{-\beta-2 \varepsilon / q}}{\left(c u^{2}-2 b u \cos \alpha_{2}+a\right)^{\lambda}} d u+\int_{1}^{\infty} \lim _{\varepsilon \rightarrow 0^{+}} \frac{u^{-\beta-2 \varepsilon / q}}{\left(c u^{2}-2 b u \cos \alpha_{2}+a\right)^{\lambda}} d u \\
\leq & \lim _{\varepsilon \rightarrow 0^{+}}\left[\int_{0}^{1} \frac{u^{-\beta-2 \varepsilon / q}}{\left(c u^{2}+2 b u \cos \alpha_{1}+a\right)^{\lambda}} d u+\int_{1}^{\infty} \frac{u^{-\beta-2 \varepsilon / q}}{\left(c u^{2}+2 b u \cos \alpha_{1}+a\right)^{\lambda}} d u\right. \\
& \left.+\int_{0}^{1} \frac{u^{-\beta-2 \varepsilon / q}}{\left(c u^{2}-2 b u \cos \alpha_{2}+a\right)^{\lambda}} d u+\int_{1}^{\infty} \frac{u^{-\beta-2 \varepsilon / q}}{\left(c u^{2}-2 b u \cos \alpha_{2}+a\right)^{\lambda}} d u\right] \\
\leq & \widetilde{C},
\end{aligned}
$$

which contradicts the fact that $\widetilde{C}<C_{\lambda}$. Hence, the constant factor $C_{\lambda}$ in (8) is the best possible.

If the constant factor in (9) is not the best possible, then by (10), we may get a contradiction that the constant factor in (8) is not the best possible. Thus the theorem is proved.

Remark 1 Setting $\lambda=a=b=c=1$ in Theorem 3.1, we have (3) and (4).

Remark 2 Setting $\lambda=1 / 2$ in Theorem 3.1, we have the following particular results:

$$
\begin{aligned}
& \int_{-\infty}^{\infty} \int_{-\infty}^{\infty} \min _{i \in\{1,2\}}\left\{\frac{1}{\sqrt{a x^{2}+2 b x y \cos \alpha_{i}+c y^{2}}}\right\} f(x) g(y) d x d y \\
& \quad<C_{1 / 2}\left(\int_{-\infty}^{\infty}|x|^{-p(\beta-1)-1} f^{p}(x) d x\right)^{1 / p}\left(\int_{-\infty}^{\infty}|y|^{q \beta-1} g^{q}(y) d y\right)^{1 / q}
\end{aligned}
$$

and

$$
\begin{aligned}
& \int_{-\infty}^{\infty}|y|^{p(1-\beta)-1}\left(\int_{-\infty}^{\infty} \min _{i \in\{1,2\}}\left\{\frac{1}{\sqrt{a x^{2}+2 b x y \cos \alpha_{i}+c y^{2}}}\right\} f(x) d x\right)^{p} d y \\
& <C_{1 / 2}^{p} \int_{-\infty}^{\infty}|x|^{-p(\beta-1)-1} f^{p}(x) d x .
\end{aligned}
$$




\section{Competing interests}

The authors declare that they have no competing interests.

\section{Authors' contributions}

Both authors contributed equally and significantly in writing this paper. Both authors read and approved the final manuscript.

\section{Author details}

${ }^{1}$ Department of Mathematical Analysis, National University of Mongolia, Ulaanbaatar, Mongolia. ${ }^{2}$ Institute of Mathematics, National University of Mongolia, P.O. Box 46A/104, Ulaanbaatar, 14201, Mongolia.

Received: 17 May 2012 Accepted: 16 January 2013 Published: 19 April 2013

\section{References}

1. Hardy, GH, Littlewood, JE, Pólya, G: Inequalities. Cambridge University Press, Cambridge (1934)

2. Mitrinović, DS, Pečarić, JE, Fink, AM: Inequalities Involving Functions and Their Integrals and Derivatives. Kluwer Academic, Boston (1991)

3. Yang, B: On Hardy-Hilbert's integral inequality. J. Math. Anal. Appl. 261, 295-306 (2001)

4. Zeng, Z, Xie, Z: On a new Hilbert-type integral inequality with the integral in whole plane. J. Inequal. Appl. 2010 Article ID 256796 (2010)

5. Xin, D, Yang, B: A Hilbert-type integral inequality in the whole plane with the homogeneous kernel of degree -2 . J. Inequal. Appl. 2011, Article ID 401428 (2011)

6. Abramowitz, M, Stegun, IA: Handbook of Mathematical Functions with Formulas, Graphs, and Mathematical Tables, 9th printing, pp. 807-808. Dover, New York (1972)

7. Azar, LE: Some extension of Hilbert's integral inequality. J. Math. Inequal. 5(1), 131-140 (2011)

8. Kuang, J: Applied Inequalities. Shangdong Science and Technology Press, Jinan (2004)

doi:10.1186/1029-242X-2013-189

Cite this article as: Adiyasuren and Batbold: On a generalization of a Hilbert-type integral inequality in the whole plane with a hypergeometric function. Journal of Inequalities and Applications 2013 2013:189.

\section{Submit your manuscript to a SpringerOpen ${ }^{\ominus}$ journal and benefit from:}

- Convenient online submission

- Rigorous peer review

- Immediate publication on acceptance

- Open access: articles freely available online

- High visibility within the field

- Retaining the copyright to your article 